\title{
Role of Magnetic Resonance Imaging in Assessment of Temporomandibular Joint Internal Derangement
}

\author{
Ismail R Atta, Medhat M. Rafaat, Ahmed E. Shalan
}

Department of radiology, Benha faculty of medicine, Benha University , Egypt

Correspondence to: Ismail R Atta, Department radiology, faculty of medicine, Benha University, Egypt

Email:

esmaeilovic@gmail.com

Received: 20 October 2019

Accepted: 12 January 2020

\begin{abstract}
Background: Magnetic resonance imaging (MRI) has become a mainstay in the evaluation of TMJ disorders given its ability of noninvasively depicting major regional anatomic structures and demonstrating the presence of joint effusion and bone marrow signal-intensity abnormalities. Consequently, MRI has become the gold standard in diagnosing articular disc displacement Aim of the study: The aim of this work was to highlight the role of magnetic resonance imaging as the gold standard imaging modality in the assessment of Temporomandibular joint internal derangement. Patients and methods: The study was performed at radiology department in Kafr El-Sheikh general hospital after obtaining written informed consent from all patients.In this study 50 patients were enrolled from the dentistry and rheumatology departments, then were referred to radiology department. Results: The overall sensitivity of MRI examination for detecting and diagnosing TMJ internal derangement was $100 \%$. Conclusion: This study suggests the use of MRI as a proper diagnostic modality for TMJ disorders due to the excellent soft tissue contrast and multiplaner capabilities that enable well demonstration of complex anatomy and well assessment of the pathological conditions of the TMJ especially internal derangement of the TMJ.
\end{abstract}

Keywords: Temporomandibular joint; internal derangement; magnetic resonance imaging 


\section{Introduction:}

Temporomandibular joint (TMJ) dysfunction is a common condition that, according to some studies, affects up to $28 \%$ of population. (1).

Magnetic Resonance Imaging is accepted as the most advanced imaging modality for diagnosis of TMJ abnormalities. It is noninvasive and has the potential to yield high quality tomographic imaging in any plane with bone as well as soft tissue spatial resolution. Additionally, the patient is not exposed to ionizing radiation or any biological hazards. Other advantages of MR imaging are its sensitivity, specificity and diagnostic accuracy (2).

The most frequent cause of TMJ dysfunction, or TMJ disorder, is internal derangement, which is defined as an abnormal relationship of the disc to the condyle. Precise localization of the disc is very important in the diagnosis of TMJ internal derangement and can easily be achieved with MR imaging. Furthermore, MR imaging help in assessment of signs of TMJ dysfunction (e.g.: joint effusion, thickening of lateral pterygoid muscle attachment \& rupture of retrodiscal layers). MR imaging also demonstrates degenerative changes (e.g.: osteoarthritis)(1).

MR imaging represents the best method for studying clinically affected joints, for evaluation of the morphological status of
TMJ and analysis of dynamic process during mouth opening. (3). This is not true dynamic images but it is pseudo dynamic MR imaging obtained from the serial multiple static images (4).

MR imaging has been shown to accurately delineate the structures of TMJ and is the best technique to correlate and compare TMJ components such as bone, disc, fluid, capsule and ligaments with autopsy specimens (5).

Since the advent of MR imaging, there have been substantial improvement in both hardware and software that currently allow better visualization of small structures such as retrodiscal layers or lateral pterygoid muscle attachment (1).

MR imaging may depict joint abnormalities not seen with any other imaging method and thus, is the best method to make a diagnostic assessment of TMJ status (6).

\section{Patients and methods:}

This study is a case control study which was performed at radiology department in Kafr ElSheikh general hospital. In this study 50 patients were enrolled from the dentistry and rheumatology departments, then were referred to radiology department after approval of the research ethical committee of Benha faculty of medicine.

The data was collected during the period of February 2019 to September 2019. 
All patients with the following clinical manifestations of TMJ dysfunction were included in this study:

- Pain in or around the TMJ during mandibular movement.

- Limited jaw opening ability.

- TMJ Locking.

- TMJ clicking sounds during mouth opening and / or closing movement.

Patients with the following manifestations were excluded from the study.

- Patient with cerebral aneurysm clip.

- Patient with cardiac pace maker.

- Patient who were claustrophobic and uncooperative.

- Metallic prosthesis heart valves, ferromagnetic foreign bodies in critical location like eye (intra-ocular foreign bodies) and cochlea (cochlear implants).

- Patient with obvious skeletal jaw deformity.

- Patient undergoing / history of orthodontic treatment.

- Patient with history of traumatic extraction.

The main tool for diagnostic assessment was the conventional MR study of the TMJ in both opened and closed mouth positions.

Conventional MR study:

All of the 50 cases have undergone routine bilateral TMJ MRI examination on 1.5 Tesla machine. Patients were asked to lay down in a supine position with both arms adducted, where the special TMJ dual coil was applied for the examination.Imaging started by axial localizer including the whole skull base. Pulse sequences were obtained from all patients in closed and maximal open mouth positions on corrected (oblique) sagittal T1 weighted; proton density (PD) and T2 weighted images with corrected (oblique) coronal views done in T1 and PD weighted images.

TMJ Localizer:

Patient position: Head first - supine, Coil type: Dual, Scan plane: Axial, Image mode: 2 dimensional (2D), Pulse sequence: Spin echo, Number of slices: 10, Slice thickness/space: 5/2mm, TR/TE: 300msec /12msec, Matrix: $256 \times 128$, Imaging time: $28 \mathrm{sec}$. The parameter for $\mathrm{T} 1$ weighted image in closed and open mouth position were as follows:-

Sagittal oblique T1 weighted image:

Patient position: Head first - supine, Coil type: Dual, Scan plane: sagittal/oblique Image mode: 2 dimensional (2D), Pulse sequence: Spin echo, Number of slices: 18, Slice thickness/space: $3 \mathrm{~mm}$ without spatial gap in between, Repetition time: 400-500 msec, Echo time: 10-20 msec, Matrix: 256 x 128, Field of view: $10-12 \mathrm{~cm}$, Imaging time: $3.14 \mathrm{~min}$.

Coronal oblique T1 weighted image:

Patient position: Head first - supine, Coil type: Dual, Scan plane: Coronal/oblique, Image mode: 2 dimensional (2D), Pulse sequence: 
Spin echo, Number of slices: 14, Slice thickness/space: $3 \mathrm{~mm}$ without spatial gap in between, Repetition time: 400-500 msec, Echo time: 10-20 msec, Matrix: 256 x 128, Field of view: $10-12 \mathrm{~cm}$, Imaging time: $3.14 \mathrm{~min}$. The parameter for PD weighted image in closed and open mouth position was as follows:-

Sagittal oblique PD weighted image:

Patient position: Head first - supine, Coil type: Dual, Scan plane: sagittal/oblique, Image mode: 2 dimensional (2D), Pulse sequence: Spin echo, Number of slices: 18, Slice thickness/space: $3 \mathrm{~mm}$ without spatial gap in between, Repetition time: 2000msec, Echo time: 10-14msec, Matrix: 256 x 128, Field of view: $10-12 \mathrm{~cm}$, Imaging time: $3.14 \mathrm{~min}$.

Coronal oblique PD weighted image:

Patient position: Head first - supine, Coil type: Dual, Scan plane: Coronal/oblique, Image mode: 2 dimensional (2D), Pulse sequence: Spin echo, Number of slices: 14, Slice thickness/space: $3 \mathrm{~mm}$ without spatial gap in between, Repetition time: $2000 \mathrm{msec}$, Echo time: 10-14msec, Matrix: 256 x 128, Field of view: $10-12 \mathrm{~cm}$, Imaging time: $3.14 \mathrm{~min}$ The parameter for $\mathrm{T} 2$ weighted image in closed and open mouth position were as follows:-

Sagittal oblique T2 weighted image:

Patient position: Head first - supine, Coil type: Dual, Scan plane: sagittal/oblique, Image mode: 2 dimensional (2D), Pulse sequence:
Spin echo, Number of slices: 18, Slice thickness/space: $3 \mathrm{~mm}$ without spatial gap in between, Repetition time: $2600 \mathrm{msec}$, Echo time: 20 msec, Matrix: 256 x 128, Field of view: $10-12 \mathrm{~cm}$, Imaging time: $3.14 \mathrm{~min}$.

Statistical analysis :

- The data collected were tabulated \& analyzed by SPSS (statistical package for social science software) statistical package version 11 on IBM compatible computer.

- Qualitative data were expressed as number and percentage (No \& \%) and analyzed by applying chi square test. Whenever the expected value in one or more of the cells in a 2X2 tables was less than 5 , fisher exact test was used instead.

- Chi square test was done for qualitative variable analysis and $\mathrm{p}$ - value $<0.05$ was considered significant.

- Sensitivity: true positive cases divided by all positive cases.

- Specificity: true negative cases divided by all negative cases.

- Accuracy: all true positive plus true negative cases divided by all cases (either true positive or true negative or false negative or false positive ).

- All these tests were used as test of significance at $\mathrm{p}<0.05$. 


\section{Results}

In this study 50 patients were included suffering from clinical manifestations of temporomandibular joint (TMJ) affections.

Table 1 shows that, out of the $50(100 \%)$ patients in the study group, $25(50 \%)$ were in the age group of 14-20 years, 18 (35.5\%) were in the age group of 21-30 years and 7 (14.3\%) were in the age group of 31-49 years. Female patients their age ranged between 14 and 48 years with a mean age of 30 years old. Male patients their age ranged between 29 and 49 years with a mean age of 35 years old. Of these 50 patients there were 33 females representing $66 \%$ of patients and 17 males representing $34 \%$ of patients.

Table (1): Statistical analysis of age and sex distribution of the examined 50 patients with clinical manifestations of temporomandibular joint affection.

\begin{tabular}{cccc} 
& \multicolumn{3}{c}{ Age } \\
& $\mathrm{N}$ & & $\%$ \\
\hline $14-20$. & 25 & & 50 \\
$21-30$. & 18 & & 36 \\
$31-49$. & 7 & & 14 \\
Total & 50 & & 100 \\
& & Sex & \\
& $\mathrm{N}$ & & $\%$ \\
Females & 33 & & 66 \\
Male & 17 & & 34 \\
Total & 50 & & 100 \\
\hline
\end{tabular}

Joint tenderness was the most frequent clinical finding (36 out of 50 patients) (72\%), followed by joint noise (32 out of 50 patients) (64\%), deflection / deviation (22 out of 50 patients) (44\%), limited mouth opening (21 out of 50 patients) (42\%), and muscle tenderness (19 out of 50 patients) (38\%) which was the least frequent clinical finding in this study as shown in table 2.

Table (2): Statistical analysis of the examined 50 patients with respect to their distribution of clinical manifestations of temporomandibular joint affection.

\begin{tabular}{lcc}
\hline Clinical symptoms & Number & Percentage \\
\hline Joint tenderness & 36 & 72 \\
Joint noise & 32 & 64 \\
Deflection / Deviation & 22 & 44 \\
Limited mouth & 21 & 42 \\
opening & 19 & 38 \\
Muscle tenderness & & \\
\hline
\end{tabular}

Figure 1 shows that, $80 \%$ of the patients suffered from bilateral joint affection and 20\% patients had unilateral joint affection. Seven of them were right sided while 3 were left sided.

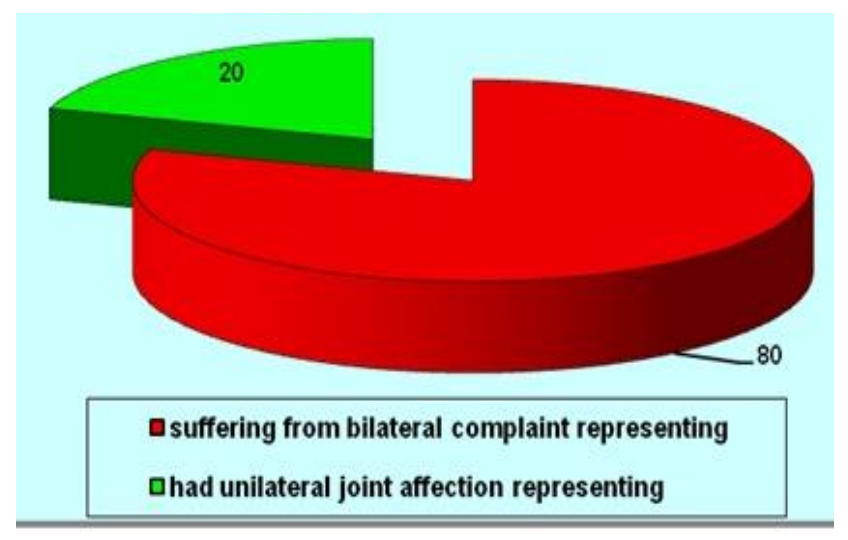

Fig. (1)

The final results of the TMJs of the studied patients examined by conventional MRI were categorized according to MR criteria into: -

A. Normal state, no disc displacement (NDD): 
Forty six out of 90 examined joints (51.1\%) had normal positioned joint. While, 25 out of the 50 examined had joints affection, where 21 of them had bilaterally affection and 4 had unilaterally affection (3 right and 1 left).This group of patients was complaining of clinical manifestations of temporomandibular joint (TMJ) affections associated with normal MR features of TMJ as regard disc shape, position and mobility. They were referred to electromyography (EMG) and nerve conduction (NC) evaluation.

Four joints show normal shape, site with loss of normal mobility i.e.: stuck disc representing 2 bilaterally affected patients and also represents $8.6 \%$ of the normally positioned discs.

Stuck disc or disc adhesion: During jaw movement, the disc remains in the same place in relation to the mandibular fossa or the articular tubercle. The stuck disc may be fixed in a normal or displaced position and may or may not be associated with normal mobility of the condyle. This is differed from normal joint state which shows in closed mouth position that the junction of the posterior band with the bilaminar zone is located above the apex of the condylar head (12 o' clock position) and when the jaw opens the condyle rotates under the disc and the disc condyle complex translates interiorly under the temporal tubercle. The disc remains interposed between the osseous component and moves anteriorly in a synchronized fashion.

Of the 4 joints with stuck discs, 2 joints showed abnormal signal representing $4.7 \%$ of the normally positioned discs.

Neither secondary changes nor joint effusion in these 46 normally positioned joints were noted.

The secondary changes of the temporomandibular joints involve degenerative osteoarthritic changes in the form of flattening, erosion, changes in the shape of the articular surfaces, anterior osteophytes and / or subchondral lacunas. (X2 5.325 P. value $\left.0.006^{*}\right)$

B. Grade I Anterior disc displacement with reduction (ADDWR):

In the closed position, the posterior band of the disc is anterior to the condylar head in all the sagittal sections. When the jaw is opened, the disc is recaptured by the condyle and the disc condyle relation appears normal.

There were 11 joints showing grade I internal derangement. They were 6 patients (5 were bilateral and 1 unilateral).

Of these 11 joints 9 joints showed normal shaped disc with normal signal and only 2 joints showed deformed discs with altered signal intensity. None of them showed secondary changes. One joint only showed 
associated effusion. (X2 4.362 P. value $0.003 *)$

C. Grade II Anterior disc displacement without reduction (ADDWOR):

In close and open mouth position, the posterior band of the disc in anterior to the superior aspect of the condylar head in all sagittal section. When the jaw is opened, the disc is anteriorly compressed, whether its shape is modified or not. They represent 19 patients (14 bilateral, 3 right and 2 left). Of these 33 joints 10 showed normal shaped disc and 23 joints show deformed disc. Also 15 of them showed altered signal while 18 of them showed normal signal. Secondary changes and effusion are only seen in 8 joints. (X2 5.257 P. value $0.009 *)$.

Grading of TMJ internal derangement:

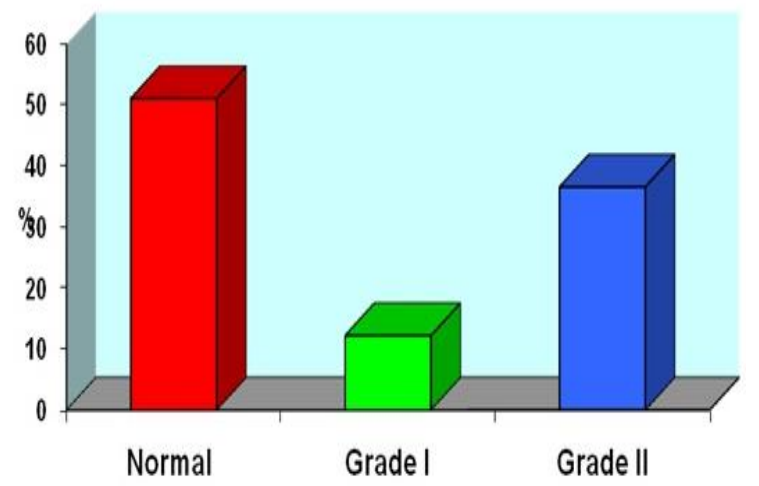

Fig (2)

-Normal state: in closed mouth position, the posterior band of the disc is located above the apex of the condylar head (12 o' clock position). When the jaw opened the intermediate zone of the disc interposed between glenoid tubercle and condylar head.

-Grade I Anterior disc displacement with reduction: in the closed position, the posterior band of the disc is anterior to the condylar head in all the sagittal sections. When the jaw is opened, the disc is recaptured by the condyle and the disc condyle relation appears as normal.

-Grade II Anterior disc displacement without reduction: in close and open mouth position, the posterior band of the disc in anterior to the superior aspect of the condylar head in all sagittal section. When the jaw is opened, the disc is anteriorly compressed, whether its shape is modified or not.

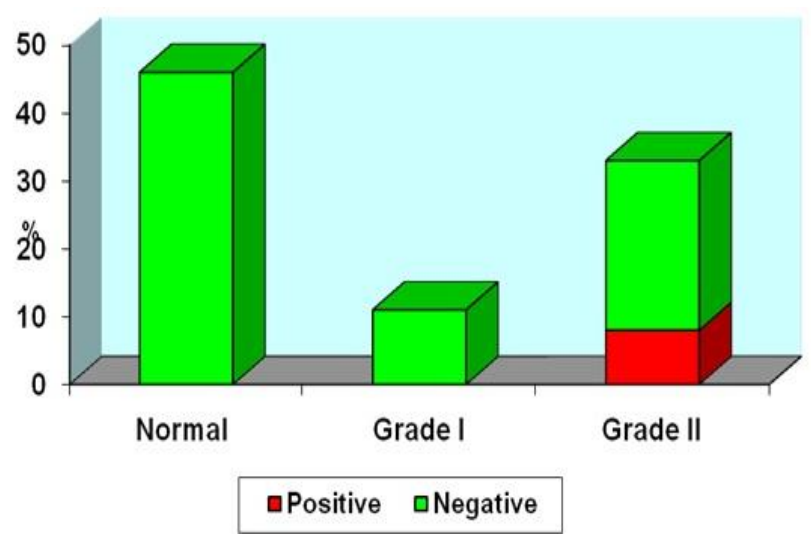

Fig (3)

Table (3): Showing disc deformity.

\begin{tabular}{cccc}
\hline & Normal & Grade I & $\begin{array}{c}\text { Grade } \\
\mathbf{I}\end{array}$ \\
\hline $\begin{array}{c}\text { Normal } \\
\text { shape }\end{array}$ & 46 & 9 & 10 \\
Deformed & 0 & 2 & 23 \\
$\mathrm{X}^{2}$ & & 3.002 & \\
\hline
\end{tabular}


-The normal configuration of the TMJ disc is biconcave configuration with both upper and lower surfaces are concave.

-The abnormal deformed shapes of the disc include biplanar shape with the disc is of even thickness; hemi convex shape with upper surface is concave and lower is convex; biconvex shape with both upper and lower surfaces are convex; folded shape with disc is folded at center .

\section{Discussion:}

Temporomandibular joint (TMJ) pain and dysfunction are important clinical problems which are commonly seen (7).

\section{Temporomandibular joint}

dysfunction is a common condition that according to some studies, affects up to $28 \%$ of population (1).

Major components of the TMJ include the mandibular condyle, the articular disc, the glenoid fossa, and the articular eminence of the temporal bone. Unlike most joints, the articulating surfaces are fibrous and not cartilaginous. The fibro cartilaginous articular disc is biconcave, dividing the joint space into superior and inferior compartments (8). With the rapid progress made in TMJ imaging techniques, many studies have focused on the importance of internal derangement, osteoarthritis (OA), effusion, and bone marrow edema (BME) as the underlying mechanisms in the etiology of TMJ disorders (9).

Internal derangement (ID) of the temporomandibular joint (TMJ) refers to the abnormal relationship or position of the disc to the mandibular condyle and articular eminence. It is considered one of the major causes of pain and dysfunction of the TMJ (10).

The current work included 50 patients with symptoms and signs of TMJ dysfunction as follows: joint pain and tenderness, joint noise and clicking sensation, limited jaw opening and muscle tenderness.

The accuracy of clinical examination for diagnosing the exact status of TMJ internal derangements has been investigated in several previous studies (11- 14), which reported that the overall accuracy of the clinical examination was $43 \%$ to $95 \%$. False positive diagnoses are related to overclassification of the disease, which might lead to over-treatment, while false negative diagnoses are related to under-classification and consequently under-treatment. The evaluation of the interobserver and interobserver agreement values revealed that disagreements in interpretation occurred mostly between the diagnoses of normal disc position and anterior disc displacement with reduction (11) 
The clinical signs and symptoms of TMJ disc derangement are not considered consistently reliable for accurate assessment of the exact extent of the internal derangement (15).

The introduction of MR imaging has expanded the diagnostic capabilities in accurate assessment of temporomandibular joint abnormalities owing to its perfect capabilities of soft tissue characterization. The use of MRI with surface coils has markedly improved the delineation of internal derangement of the TMJ and has expanded the knowledge of the anatomical details of such joint enhancing the capabilities of the diagnosis of certain pathological processes involving this joint. It clarified also the relationship of certain MRI findings and patients symptoms (16).

In the present work, sex showed statistically significant differences between the studied group. Out of the 50 studied patients, 33 were females \& 17 were males. The ratio of female to male in this group of patients with temporomandibular joint disorders was 1.9:1. The results of this study show that the prevalence of temporomandibular joint disorder was higher in women than in men. This is in agreement with Amin et al study, (10) who found increased ratio of female to male patients with TMDs (2.5:1). Several related studies were reported by Okeson et al (17) with comparable results.
In another study done by Dalkiz et al (18) showed that pain and dysfunction TMJ disorders seem to affect women more than men with clinical reports have emphasized the high ratio $(8: 1)$ of female to male patients for TMJ disorders.

The factors responsible for this predominance are not known (15).However, It has been suggested in animal studies that joint laxity involving any joint occurs more commonly in females than in males. These findings may represent a sex-limited expression of joint laxity that is related to altered collagen synthesis, which is affected either by the level of circulating estrogen or the concentration of estrogen receptors within joints. It is suggested that individuals with joint laxity as a result of altered collagen synthesis are at greater risk of developing bilateral TMD when subjected to etiological factors such as trauma, joint overextension, or joint overuse (19).

In this study, the most common clinical sign was pain/tenderness in preauricular region (36 out of 50 patients $72 \%$ ).

Pain is a symptom which arises frequently in cases of TMJ pathologies. With regards to the patients who suffer TMJ pain, numerous studies based on the examination of the MR findings have been carried out (20).

On the contrary, Adame et al (21) in their study stated that the cause of pain/ tenderness 
could be associated with retrodiscal tissue alteration, capsulitis, synovitis. This is in agreement with a study done by Farina et al (22) showed that the MR signal changes in the retrodiscal tissue are highly correlated with TMJ pain.

In our study, bilateral affection of the temporomandibular joints was noted in 40 patients representing $80 \%$ of the examined patients. Bilateral internal derangement has been demonstrated in $79 \%$ of patients in a study comprising 96 joints done by Wik et al (23) and another study (24) obtained similar results by using MRI to examine both TMJs in every patient with signs and symptoms of TMJ internal derangement whether unilateral or bilateral.

In the study done by Romanelli et al (25) bilateral temporomandibular joints affection was noted in $63 \%$ of cases examined with MRI. It is suggested that the incidence of bilateral internal derangement in patients may be in the range of $50 \%$ to $60 \%$. The reason for the high incidence of bilateral involvement of the TMJs is unknown. However, it may be hypothesized that patients who have a prior history of injury or trauma to the head, neck or jaws may have sustained either direct or indirect damage to the TMJ. Therefore, any exogenous factor that may participate in the development of internal derangement in one temporomandibular joint may lead to development of internal derangement in the contralateral joint. While symptoms may be present in both joints, patient may experience pain and dysfunction of greater intensity and severity on the other side (25).

In the present study the incidence of disc displacement was $48.8 \%$ compared to $54 \%$ in another study (26).

In a study done by Amin et al (10) on 28 joints examined by MRI, 8 joints showed disc displacement with reduction (28.7\%), while 16 joints showed disc displacement without reduction $(57.1 \%)$.

In our study there was 11 joints showing anterior disc displacement with reduction representing $12 \%$ of cases and there was 33 joints showed anterior displacement without reduction representing $36 \%$ of the examined joints.

In a study done by Yilmaz and Toller (27) after evaluating 133 temporomandibular joints by MRI; they found that the articular disc is deformed in $52.38 \%$ of the joints with reduced anteriorly displaced disc whereas $85.71 \%$ of the non-reducible discs were deformed.

In the present study the deformed discs represent $70 \%$ of the anteriorly displaced non reducible. In the anteriorly displaced reducible discs, disc deformities represented $18 \%$. 
The close association between permanent disc displacement (without reduction) and bone changes is well documented (28).

Larheim et al (29) and Sano et al. (30) reported that the degree of pain in TMJs with bone marrow abnormalities was significantly higher than in TMJs with normal bone marrow signal on MR images which supports the concept that bone marrow abnormalities represent a response to an increased intraarticular pressure in conditions of pronounced inflammatory process such as synovitis.

In our study we have diagnosed $46(51.1 \%)$ TMJs showing normally positioned articular disc out of the 90 examined joints. The remaining 44 joints showed anterior disc displacement representing $48.9 \%$ of all examined joints. Of the 46 normally positioned discs, 4 discs were stuck representing $8.6 \%$ and 42 discs were mobile. The 44 displaced discs were all mobile with no stuck discs in this group. This is in agreement with another study (10); they found that stuck disc represent $10.7 \%$ of the studied group. On the contrary, in a study done by Rao et al (31), they reported 127 joints with normal disc position in the closed mouth out of 212 TMJs in 106 patients representing $60 \%$ of the examined joints. The articular disc was displaced in 85 joints (40\%). Of the 127 joints with normally positioned discs, $94(80 \%)$ had mobile discs and 23 (20\%) had stuck discs. The remaining 10 joints were excluded since there was no condylar translation and therefore the disc mobility could not be assessed. Of the 85 joints with displaced discs, 67 (82\%) had mobile discs and 15 (18\%) had stuck discs.

Our results would have been more representative if we had a larger study population, so we could have caught more number of cases with abnormal findings especially those involving the outflow tracts.

- It is recommended to conduct studies on a larger scale of patients complaining of TMJ disorders in order to investigate other types of TMJ displacement either sideway or rotational displacements and other TMJ pathologies responsible for patients clinical signs and symptoms.

- Also, it is recommended to apply dynamic (kinematic) MRI assessment of the TMJ when it is clinically needed to assess the biomechanics of the joint and in patients with normal static MRI in spite of clinical signs and symptoms.

\section{Conclusion:}

This study suggests the use of MRI as a proper diagnostic modality for TMJ disorders due to the excellent soft tissue contrast and multiplaner capabilities that enable well demonstration of complex anatomy and well assessment of the pathological conditions of 
the TMJ especially internal derangement of the TMJ.

\section{Conflict of interest: None declared}

\section{References:}

1. Tomas X, Pomes J, and Berenguer J.: MRI imaging of temporomandibular joint dysfunction: a pictorial review. Radiographic 2006; 26(3):765-81.

2. Michelle A, Wessely F, and Martin Y.: Magnetic resonance imaging of the temporomandibular joint. Clinical chiropractic 2008; 11:37-44.

3. Mark S, Bodo $\mathrm{K}$, and Christina L.: Temporomandibular joint disc position assessed at coronal MR imaging in asymptomatic volunteers. Radiology 2005; 236:559-564.

4. Jin-HO L , Kyoung-In Y , and In-Woo P.: Comparison of static MRI and Pseudo-dynamic MRI in temporomandibular joint disorder patients. Korean journal of oral and maxillofacial radiology 2006; (36):199-206.

5. Rudisch A, Emshoff R, Maurer H, Kovacs $\mathrm{P}$, Bodner G..: Pathologic-sonographic correlation in temporomandibular joint pathology. Eur Radiol 2006; 16: 1750-1756.

6. Peterová V, Jirman R, and Mazánek J.: The examination of the temporomandibular joint on 1,5T magnetic resonance. Prague medical report 2004; 105(1):29-34.

7. Tasali N, Cubuk R, Aricak M, Ozarar M, Saydam B, Nur H.et al.: Temporomandibular joint (TMJ) pain revisited with dynamic contrast-enhanced magnetic resonance imaging (DCE-MRI). European Journal of Radiology 2011; 5197:6.

8. Wang EY, and Fleisher KA.: MRI of temporomandibular joint disorders. Applied radiology 2008; 17-24.

9. EMSHOFF R, BRANDLMAIER I, GERHARD S, STROBL H, BERTRAM S, RUDISCH A..: Magnetic resonance imaging predictors of temporomandibular joint pain. J Am Dent Assoc 2003; 134:705-14.

10. Amin M , Hassan A and Barakat K.: The accuracy of dynamic Magnetic resonance imaging in evaluation of internal derangement of the temporomandibular joint; comparison with arthroscopic findings. The Egyptian Journal of Radiology and Nuclear Medicine 2012; 43: 429436.

11. Kobs G, Bernhardt $\mathrm{O}$ and Meyer G.: Magnetic resonance evaluation between the relationship of the temporomandibular joint disc and condylar head displacement. Stomatologija, Baltic Dental and Maxillofacial Journal 2003; 5:93-6.

12. Foucart JM, Carpentier P, Pajoni D, MarguellesBonnet R, Pharaboz C..: Magnetic resonance imaging of 732 temporomandibular joints: Anterior, rotational, partial and sideways disc displacement. European J of Radiology 1999; 28:385-94.

13. Barclay P, Hollender LG, Maravilla KR, Truelove EL..: Comparison of clinical and magnetic resonance imaging diagnoses in patients with disc displacement in the temporomandibular joint. E.L, Oral Surg Oral Med Oral Pathol Oral Radiol Endod 1999; 88:37-43.

14. Del-Balso AM.: An approach to the diagnostic imaging of jaw lesions, dental implants and the temporomandibular joint. Radiol Clin North Am 1998; 36(5):855-90.

15. Katzberg W.: Temporomandibular joint imaging. Radiology1998; 170:297-307.

16. El-Essawy M, Al-Nakshabandi $\mathrm{N}$ and Al-Boukai A.: Magnetic resonance imaging evaluation of temporomandibular joint derangement in symptomatic and asymptomatic patients. Saudi Med J 2008; 29(10): 1448-52.

17. Okeson JP.: Diagnosis of temporomandibular disorders in management of temporomandibular disorders and occlusion. St Louis, Missouri: Mosby 2008; pp. 285. 
18. Dalkiz M, Pakdemirli E and Beydemir B.: Evaluation of TMJ dysfunction by Magnetic resonance imaging. Turk J Med Sci 2001; 31: 337 343.

19. Nebbe B and Major PW.: Prevalence of TMJ disc displacement in a pre-orthodontic adolescent sample. Angle orthodontist 2000; 70: No 6.

20. Chiba M, Kumagai $M$ and Echigo S.: Assocition between high signal intensity in the posterior disc attachment seen on T2 weighted fat-supressed images and temporomanibular joint pain. Dentomaxillofac radiol 2007; 36(4):187-91.

21. Adame CG, Monje F, Offnoz M and Mrtin-Granizo R.: Effusion in magnetic resonance imaging of the temporomandibular joint: a study of 123 joints. J Oral Maxillofac Surg 1998; 56:314-8.

22. Farina D, Bodin C, Gandolfi S, De Gasperi W, Borghesi A, Maroldi R..: TMJ disorders and pain: assessment by contrast-enhanced MRI. Eur J Radiol 2009; 70:25-30.

23. Wilk RM, Harms SE and Wolford LM.: Magnetic resonance imaging of the temporomandibular joint using a surface coil. J Oral Maxillofac Surg 1986; 44:935-43.

24. Sanchez-Woodworth RE, Tallents RH, Katzberg RW, Guay JA..: Bilateral internal derangements of the temporomandibular joint: Evalution by magnetic resonance imaging. Oral Surg Oral Med Oral Pathol 1988; 65:281-5.

25. Romanelli GG, Harper R, Mock D, Pharoah MJ, Tenenbaum HC..: Evaluation of temporomandibular joint internal derangement. J Orofac Pain 1993; 7(3):254-62.

26. Maizlin Z, Nutiu N, and Dent P.: Displacement of the temporomandibular joint disc: correlation between clinical findings and MRI characteristics. Applied research 2010; 76:a3.

27. Yilmaz NT and Toller MO.: Magnetic resonance imaging evaluation of temporomandibular joint disc deformities in relation to type of disc displacement. $\mathrm{J}$ Oral Maxillofac Surg 2001; 59:860-865.

28. Milano V, Desiate A, Bellino R, Garofalo T..: Magnetic resonance imaging of temporomandibular joint disorders: classification, prevalence and interpretation of disc displacement and deformation. Dent Maxillofac Rad 2000; 29:352-61.

29. Larheim TA, Katzberg RW, Westesson PL, Tallents $\mathrm{RH}, \quad$ Moss ME..: MR evidence of temporomandibular joint fluid and condyle marrow alterations: occurrence in asymptomatic volunteers and symptomatic patients. Int $\mathbf{J}$ Oral Maxillofac Surg $2001 ; 30: 113-117$.

30. Sano T, Westesson PL, Larheim TA and Takagi R.: The association of temporomandibular joint pain with abnormal bone marrow in the mandibular condyle. J Oral Maxillofac Surg 2000; 58:254-7.

31. Rao VM, Liem MD, Farole A, Razek AA..: Elusive stuck disc in the temporomandibualr joint diagnosis with MR imaging. Radiology 1993; 189:823-827.

To cite this article: Ismail R Atta, Medhat M. Rafaat, Ahmed E. Shalan. Role of magnetic resonance imaging in assessment of temporomandibular joint internal derangement, BMFJ, 2020; 37(2): 369-381. DOI: 10.21608/bmfj.2020.18445.1110 